\title{
Cutaneous and pharyngeal diphtheria imported from the Indian subcontinent
}

\author{
PE Hart, PYC Lee, DC Macallan, MH Wansbrough-Jones
}

\begin{abstract}
Summary
We report the case of a 32-year-old woman who presented upon returning from India with cutaneous ulcers on the feet and pharyngitis. Microbiological testing showed the causative organism to be a toxigenic strain of Corynebacterium diphtheriae. She was treated successfully with penicillin and diphtheria antitoxin. This case emphasises the importance of maintaining a high index of suspicion for such rare but significant infectious diseases.
\end{abstract}

Keywords: diphtheria, Corynebacterium diphtheriae, toxigenic

Corynebacterium diphtheriae was first described as the cause of diphtheria by Loeffler in 1884 . At that time it ravaged most of the world, and was known as 'the strangling angel of children'. With the advent of widespread vaccination, it was hoped that this disease would fade into obscurity. Instead, it remains a significant health problem in many parts of the world, and the developed countries are not immune to its threat.

Since the introduction of immunisation against diphtheria in the $1940 \mathrm{~s}$, its incidence has declined rapidly, to the extent that it is considered eliminated in the immunised population of industrialised countries, with toxigenic strains no longer circulating among the native population. ${ }^{1}$ This reduced prevalence results in reduced awareness. However, diphtheria is endemic in many developing countries and epidemics occur, most recently in Russia. ${ }^{2}$

Diphtheria typically presents as a severe pharyngitis with membrane formation, which may extend to the larynx and trachea causing respiratory compromise. The toxin liberated can cause myocardial and neurological damage. Diphtheria may also occur as a cutaneous form, and this acts as an important source for the dissemination of the infection. ${ }^{3}$ Other forms that diphtheria may take include endocarditis, septic arthritis, splenic abscess, or bacteraemia.

Vaccination substantially reduces the risk of developing the complications of diphtheria, but serological surveys have shown that many people in the developed world are not protected. ${ }^{4,5}$ In a survey of over 3000 Italian army recruits the level of non-protection was $22.9 \%$, compared with $5.3 \%$ for tetanus. ${ }^{6}$ These factors, combined with the increasing ease of world travel, make the importation of diphtheria ever more likely. We now report such a case.

\section{Case report}

A 32-year-old Caucasian woman presented 10 days after returning from a one-month holiday in India. Three weeks previously she had developed an abrasion in the first toe space of the right foot. At first she attributed this to trauma from her footwear, but it later developed into a true ulcer. Two weeks later a similar lesion appeared on her left foot. Corynebacterium diphtheriae was subsequently cultured from microbiological swabs of these lesions taken by her general practitioner.

At the time of admission she reported a sore throat for two days, and generalised myalgia for one day. She had received full childhood vaccination including diphtheria toxoid. On examination she was apyrexial. There were two ulcers on the feet (figure 1). The pharynx was inflamed with a firmly adherent exudate over the left posterior pharyngeal wall (figure 2) with posterior cervical lymphadenopathy. No other abnormalities were present.

Corynebacterium diphtheriae var mitis was cultured from throat swabs. The organism proved to be toxin positive, and this was confirmed by polymerase chain reaction for the toxin gene. ${ }^{7}$ Full blood count was normal, C-reactive protein was $147 \mathrm{mg} / \mathrm{l}$, erythrocyte sedimentation rate $23 \mathrm{~mm} / \mathrm{h}$. Electrocardiogram was normal. Standard respiratory isolation measures were enforced. Treatment was commenced with intravenous benzyl penicillin, 2 megaunits four hourly, and after administration of a test dose, 40000 units of diphtheria antitoxin. She made an uneventful recovery. The patient's husband, who had travelled with her in India but remained well, received diphtheria booster vaccination and erythromycin orally.

\section{Discussion}

This case illustrates the occurence of toxigenic $C$ diphtheriae infection in a traveller returning to the UK. Clinical suspicion must be high to include diphtheria in the differential diagnosis of sore throat or skin ulcer in a traveller. A full travel history is essential, as is the need to take the appropriate microbiological samples. Furthermore, once the samples arrive in the laboratory, the correct cultures need to be set up. This was done by the laboratory techni- 


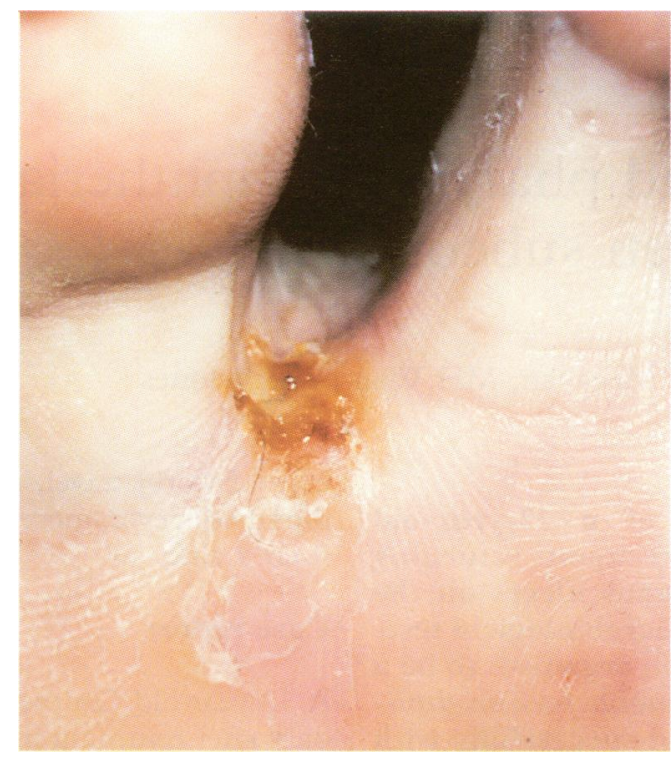

Figure 1 Cutaneous diphtheria lesion in the first toe web space

cian, relying on the travel history provided by the general practitioner.

The value of polymerase chain reaction in the detection of toxigenic strains has previously been documented. ${ }^{8}$ The main benefit is that it drastically reduces the time taken to prove that the organism is a toxin-producing strain, and may therefore allow treatment to be instituted earlier.

Cutaneous diphtheria is not a notifiable disease, even though it may be caused by toxigenic strains. It may lead to pharyngeal involvement by a process of autoinfection, and we believe that this was the sequence of events in this case. Cutaneous diphtheria should be considered in the differential diagnosis of atypical skin infections in travellers returning from abroad, and can be identified by appropriate culture techniques.

The absence of systemic toxicity in this case may be related to the slow release of toxin from

1 Karzon DT, Edwards KM. Diphtheria outbreaks in immunized populations. N Engl f Med 1988; 318: $41-3$ immunized populations. N Engl f Med 1988; 318: 41-3 MMWR 1993; 42: 840-41, 847.

$M M W R$ 1993; 42: 840-41, 847.
Jamlin C, Stuart GH. Cutaneous diphtheria in Bristol. 3 Jamlin C, Stuart GH. Cutaneous

4 Bowler ICJ, Mandal BK, Schlecht B, Riorden T. Diphtheria, the continuing hazard. Arch Dis Child 1988; 63: 194210.

5 Farizo KM, Strebel PM, Chen RT, Kimbler A, Cleary TJ, Cochi SL. Fatal respiratory disease due to Corynebacterium diphtheria: case report and review of guidelines for management, investigation and control. Clin Infect Dis 1993; 16: $59-68$.

6 Rappouli R, Podda A, Giovannoni F, Nencioni L, Peragallo M, Francolini $P$. Absence of protective immunity against diphtheria in a large proportion of young adults. Vaccine 1993; 11: $576-7$.

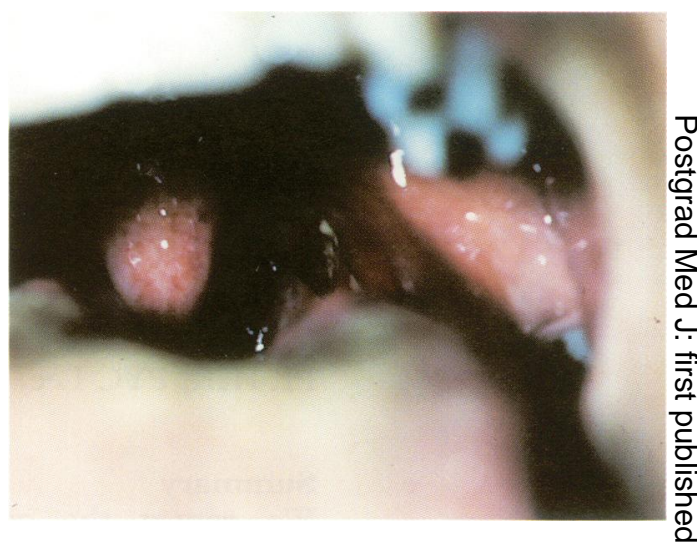

Figure 2 Early diphtheria membrane overlying the left palatal tonsil

\section{Learning points}

- diphtheria is an important infectious disease with serious potential complications

- despite intense vaccination programmes many individuals have inadequate immunity

- there are many areas in the world where diphtheria is still endemic

- diphtheria may present in atypical forms, such as the cutaneous variety

- toxigenicity can be rapidly assessed by polymerase chain reaction techniques

the initial cutaneous site, thus allowing time for a protective immune response to develop. Also previous vaccination may have reduced the severity of systemic reactions. ${ }^{9}$ Of equal im- $\frac{\infty}{\Phi}$ portance to treatment of the case is the implementation of control measures for preventing the spread of toxigenic $C$ diphtheriae. This includes taking nose and throat swabs from close contacts for isolation, giving antibiotic prophylaxis, and giving all close contacts 3 . a full primary course of immunisation or a $\dot{0}$ booster dose, depending on their immunisation history.

7 Pallen MJ, Hay AJ, Puckey LH, Efstratiou A Polyn corynebac- 7 teria for the production of diphtheria toxin. $f$ Clin Pathol 994; 47: 353-6.

8 Pallen MJ. Detection and characterisation of toxin genes $\sigma$ and insertion sequences by PCR and other molecular $N$ techniques. MD Thesis, University of London, 1993; pp $35-48$

9 Benenson AS, ed Control of communicable disease in man. 15th edn. American Public Health Association, 1990; pp $138-142$. 